\title{
Biosorption and biodegradation of polycyclic aromatic hydrocarbons by Phanerochaete chrysosporium in aqueous solution
}

\author{
DING Jie ${ }^{1,2}$, CHEN BaoLiang ${ }^{1,2 *} \&$ ZHU LiZhong ${ }^{1,2}$ \\ ${ }^{1}$ Department of Environmental Science, Zhejiang University, Hangzhou 310058, China; \\ ${ }^{2}$ Zhejiang Provincial Key Laboratory of Organic Pollution Process and Control, Hangzhou 310058, China
}

Received March 30, 2012; accepted May 21, 2012; published online August 16, 2012

\begin{abstract}
Biosorption and biodegradation of phenanthrene and pyrene by live and heat-killed Phanerochaete chrysosporium are investigated to elucidate the bio-dissipation mechanisms of polycyclic aromatic hydrocarbons (PAHs) in aqueous solution and its regulating factors. The effects of nutrient conditions (carbon source and nitrogen source concentrations), the co-existing $\mathrm{Cu}^{2+}$, and repeated-batch feed of PAHs on the biosorption and biodegradation are systematically studied. The removal of PAHs by dead bodies of $P$. chrysosporium is attributed to biosorption only, and the respective partition coefficients of phenanthrene and pyrene are 4040 and $17500 \mathrm{~L} / \mathrm{kg}$. Both biosorption and biodegradation contribute to the dissipation of PAHs by live P. chrysosporium in water. After a 3-d incubation, the removal percentage via biosorption are $19.71 \%$ and $52.21 \%$ for phenanthrene and pyrene, respectively. With the increase of the incubation time (3-40 d), biodegradation gradually increases from $20.40 \%$ to $60.62 \%$ for phenanthrene, and from $15.55 \%$ to $49.21 \%$ for pyrene. Correspondingly, the stored-PAHs in the fungal bodies decrease. Under the carbon-rich and nitrogen-limit nutrient conditions, the removal efficiency and biodegradation of phenanthrene and pyrene are significantly promoted, i.e. $99.55 \%$ and $92.77 \%$ for phenanthrene, and $99.47 \%$ and $83.97 \%$ for pyrene after a 60 -d incubation. This phenomenon is ascribed to enhanced-biosorption due to the increase of fungal biomass under carbon-rich condition, and to stimulated-biodegradation under nitrogen-limit condition. For the repeated-batch feed of phenanthrene, the pollutant is continuously removed by live $P$. chrysosporium, and the contribution of biodegradation is enhanced with the repeated cycles. After 3 cycles, the biodegradation percentage is up to $90 \%$ with each cycle of a 6 -d incubation.
\end{abstract}

biosorption, biodegradation, Phanerochaete chrysosporium, polycyclic aromatic hydrocarbons, bioremediation

Citation: Ding J, Chen B L, Zhu L Z. Biosorption and biodegradation of polycyclic aromatic hydrocarbons by Phanerochaete chrysosporium in aqueous solution. Chin Sci Bull, 2013, 58: 613-621, doi: 10.1007/s11434-012-5411-9

Persistent organic pollutants (POPs) constitute a diverse group of organic substances, which are persistent, toxic, bioaccumulative and prone to long-range transport [1-3]. POPs are widely distributed in the environment $[1,4,5]$, which are harmful to human health by breathing, drinking and food chain [6]. Therefore, the interfacial behavior, environmental transport, health effects, pollution control and restoration of POPs are widely concerned [6-10]. Microbial remediation is one of the most potential environmentally-friendly technology to treat POPs contaminated sites [10-15].

In the process of bioremediation, microorganisms can be

*Corresponding author (email: blchen@zju.edu.cn) viewed both as a bio-sorbent that accommodates organic pollutants and as a bioreactor that degrades them [15]. Biosorption is a physic-chemical process involved in the sorption of a chemical substance in/on a biological matrix/ surface $[16,17]$. So far, the biosorption of heavy metal, dyes, and pesticides by biological materials such as bacteria, fungus and algae were extensively reported [16-20], but the biosorption of POPs has received considerably less attention. Inactivated biological materials were usually chosen for actual pollution control because the activity of live microorganisms is affected by nutritional elements, $\mathrm{pH}$ and other conditions [19-21]. Ding et al. [22] reported that the biosorption mechanism of phenanthrene and pyrene by dead white-rot fungi was dominated by partition into fungal bio- 
mass and biosorption-desorption was reversible. Most studies about bioremediation are focused on biodegradation of organic pollutants; however, the biosorption of soil indigenous microbial body and their impact on biodegradation are still unknown, because it is difficult to distinguish biosorption of microbial body and sorption of soil in the complex system [15].

Recently, the influences of biosorption and biodegradation on the transport, fate and pollution control of POPs in the environment have been increasingly concerned. Stringfellow and Alvarez-Cohen [23] suggested that biosorption can decrease the rate of polycyclic aromatic hydrocarbons (PAHs) biodegradation in short term, and contributed to the immediate removal of PAHs from polluted solution. Chen et al. [15] quantitatively described the contribution of biosorption and biodegradation to the total bio-dissipation of PAHs, and suggested that the ratio of apparent partition coefficients in live fungal systems $\left(K_{\mathrm{d}}{ }^{*}\right)$ to the partition coefficient of heat-killed fungi $\left(K_{\mathrm{d}}\right)$ can be employed to illustrate the relative contributions of biosorption and biodegradation under different nutrient conditions. Biosorption reduced biodegradation percentage, while nitrogen limitation and co-existing of PAHs improved biodegradation. Yesilada et al. [24] compared the decolorization capability of viable pellets with that of dead pellets during repeated batch studies, and indicated that the decolorization of the dead pellets gradually decreased while the viable showed an obviously higher decolorization. The combined contribution of biosorption and biodegradation resulted in the higher pollution removal. However, the regulating principles and enhanced approaches of biosorption and biodegradation of POPs during repeated batch process are still wanted.

Phanerochaete chrysosporium, a typical white-rot fungus is capable to degrade lignin-like polycyclic compounds such as PAHs $[25,26]$. White-rot fungi are widespread concern in the microbial remediation of POPs contaminated environment [27-30] because they can use multiple co-metabolic substrates [27,28], easily to grow and are adaptable to different environmental conditions. PAHs are a typical class of persistent organic pollutants. To precisely predict transport and fate of PAHs in the environment co-existing with microorganisms and provide a fundamental gist for bioremediation, the relative contribution of biosorption and biodegradation of PAHs by live and heat-killed $P$. chrysosporium and the influence of carbon source, nitrogen source and co-existing $\mathrm{Cu}^{2+}$ were studied. In addition, the biosorption and biodegradation of repeated-batch feed of PAH were also investigated.

\section{Materials and methods}

\subsection{Experimental materials}

Phenanthrene (Phen, $\mathrm{C}_{14} \mathrm{H}_{10},>97 \%$ ) and pyrene (Pyr,
$\mathrm{C}_{16} \mathrm{H}_{10},>98 \%$ ) were purchased from Sigma-Aldrich Corporation. The respective molecular mass of Phen and Pyr are 178.2 and 202.3. The individual aqueous solubilities are 1.18 and $0.13 \mathrm{mg} / \mathrm{L}\left(25^{\circ} \mathrm{C}\right)$. The octanol-water partition coefficients are $2.8 \times 10^{4}$ and $8.0 \times 10^{4}$, respectively. $P$. chrysosporium chosen in the experiment was bought from China General Microbiological Culture Collection Center, and the CGMCC number is 5.0776. Culture medium (Martin broth, a selective medium for fungi) was made by 0.5 $\mathrm{g} / \mathrm{L} \mathrm{MgSO}_{4} \cdot 7 \mathrm{H}_{2} \mathrm{O}, 1 \mathrm{~g} / \mathrm{L} \mathrm{KH}_{2} \mathrm{PO}_{4}, 10 \mathrm{~g} / \mathrm{L}$ glucose, $5 \mathrm{~g} / \mathrm{L}$ peptone and $16 \mathrm{~g} / \mathrm{L}$ agar in $1 \mathrm{~L}$ of distilled-deionized water for plate culture. After a 5 -d incubation at $35 \pm 0.5^{\circ} \mathrm{C}$, the culture plate was uniformly covered with colonies, and then the spore of $P$. chrysosporium was harvested by washing with sterile water. The spore suspension of $10^{5}$ ceus $/ \mathrm{mL}$ was inoculated to $150 \mathrm{~mL}$ liquid culture solution and was incubated for another $7 \mathrm{~d}$ at $35 \pm 0.5^{\circ} \mathrm{C}$ and $120 \mathrm{r} / \mathrm{min}$. The mycelial pellets with similar size (about $0.6 \mathrm{~cm}$ ) were selected for further experiments.

\subsection{Biosorption and biodegradation experiments of Phen and Pyr by $P$. chrysosporium pellets in solution}

Both live and dead mycelial pellets were used in biosorption and biodegradation experiments. The dead mycelial pellets were obtained by autoclaving live mycelial pellets under $121^{\circ} \mathrm{C}$ for $20 \mathrm{~min}$. Two treatments were prepared in microcosmic systems of 40-mL vials by adding a grain of living or dead mycelia pellets. After the addition of $15-\mathrm{mL}$ aqueous solution with the concentrations of $1.0 \mathrm{mg} / \mathrm{L}$ Phen and $0.1 \mathrm{mg} / \mathrm{L} \mathrm{Pyr}$, the vials were sealed with aluminum foillined Teflon screw caps to avoid evaporation, and then agitated in the dark at $35 \pm 0.5^{\circ} \mathrm{C}$ with aeration for 2 min each day. The upper part of vials left enough atmosphere to support the growth of mycelial pellets. Twelve replicate samples were prepared for each group, and three samples were measured at the incubation time of 3-, 7-, 14-, and 40-d. The solution was separated from solids by centrifugation at 3000 $\mathrm{r} / \mathrm{min}$ for $10 \mathrm{~min}$. The residual PAHs in solution and mycelial pellets were examined. The supernatant was removed and diluted with acetonitrile $(1: 1, \mathrm{v}: \mathrm{v})$, and the residual PAHs in the solution were detected. Then the solution in the vials was decanted as much as possible, and the residual PAHs in mycelial pellets were extracted by ultrasonication for $30 \mathrm{~min}$ with a $10 \mathrm{~mL}$ mixture of acetone and hexane $(1: 1, v: v)$ for 3 successive extractions. The extracts were pooled and evaporated to almost dryness using a rotary evaporator and re-dissolved in $5 \mathrm{~mL}$ of hexane, followed by a clean-up procedure through a 2.5 -g silica gel column with a $15 \mathrm{~mL}$ mixture of hexane and dichloromethane $(1: 1, \mathrm{v}: \mathrm{v})$. Samples were evaporated again and re-dissolved in acetonitrile to a final volume of $4 \mathrm{~mL}$. After filtration through 0.22 $\mu \mathrm{m}$ filter units, PAHs concentrations were determined by a high performance liquid chromatography (HPLC, Agilent 1200) with a fluorescence detector (FLD) using acetonitrile- 
water $(90: 10, \mathrm{v}: \mathrm{v})$ as the mobile phase at a flow rate of 1 $\mathrm{mL} / \mathrm{min}$ at column temperature $30^{\circ} \mathrm{C}$. The individual excitation wavelengths of Phen and Pyr were 244 and $237 \mathrm{~nm}$, while the emission wavelengths were 360 and $385 \mathrm{~nm}$, respectively. The vials with mycelial pellets were oven-dried at $65^{\circ} \mathrm{C}$ for $8 \mathrm{~h}$, and the dry weight of mycelial pellets was measured by mass difference. A blank, containing PAHs solution without a mycelia pellet was also prepared as a control for evaporation loss. To measure the biosorption and biodegradation of PAHs in solution, the biosorption percentage $(S)$, degradation percentage $(D)$ and removal percentage $(R)$ were calculated by

$$
\begin{gathered}
R=\left(C_{0}-C_{\text {soln }}\right) / C_{0} \times 100 \%, \\
S=m_{\mathrm{p}} /\left(C_{0} V\right) \times 100 \%, \\
D=(R-S) \times 100 \%,
\end{gathered}
$$

where $C_{0}(\mu \mathrm{g} / \mathrm{mL})$ is the initial concentration of PAHs, $C_{\text {soln }}$ $(\mu \mathrm{g} / \mathrm{mL})$ is the aqueous concentration of PAHs at different incubation time, $m_{\mathrm{p}}(\mu \mathrm{g})$ is the mass of PAHs that sorbed by mycelial pellets, and $V(\mathrm{~mL})$ is the volume of solution.

\subsection{Influence of carbon source, nitrogen source and the concentration of spore suspension}

Carbon and nitrogen are the necessary nutrient elements for fungi and control the enzyme activity of $P$. chrysosporium $[31,32]$, so that these two nutrient elements are critical parameters to affect biosorption and biodegradation of PAHs. Glucose is the most common carbon source, while $P$. chrysosporium is insensitive to the types of nitrogen source [33]. A modified Martin broth was used in the experiment, which contained $0.5 \mathrm{~g} / \mathrm{L} \mathrm{MgSO} 4 \cdot 7 \mathrm{H}_{2} \mathrm{O}, 1 \mathrm{~g} / \mathrm{L} \mathrm{KH}_{2} \mathrm{PO}_{4}$ in 1 $\mathrm{L}$ of distilled-deionized water, glucose as the carbon source, sodium nitrate as the nitrogen source, and the $\mathrm{pH}$ of culture within $4.5 \pm 0.1$. As shown in Table 1 , the concentrations of glucose were $5.6 \mathrm{mmol} / \mathrm{L}$ for carbon-limited groups and 56 $\mathrm{mmol} / \mathrm{L}$ for carbon-rich groups while the concentrations of sodium nitrate were $2.4 \mathrm{mmol} / \mathrm{L}$ for nitrogen-limited groups and $24 \mathrm{mmol} / \mathrm{L}$ for nitrogen-rich groups [33]. The selected concentrations of spore suspension were $10^{5}$ and $10^{6}$ ceus/ $\mathrm{mL}$. The influences of carbon source, nitrogen source and concentrations of spore suspension on biosorption and biodegradation of PAHs were determined by a $\mathrm{L}_{8}\left(2^{7}\right)$ orthogonal test. As shown in Table 1, after 1.0-mL spore suspension, 20-mL aqueous solution with the concentrations of 1.0 $\mathrm{mg} / \mathrm{L}$ Phen and $0.1 \mathrm{mg} / \mathrm{L}$ Pyr were added into a $40-\mathrm{mL}$ vial. The residual PAHs in solution and in the fungal biomass, and the dry weight of fungi were measured at the incubation time of 10- and 60-d. A group with $\mathrm{NaN}_{3}$ as a bacteriostat was set as a blank control.

\subsection{Experiment of biosorption and biodegradation of Phen in the repeated batch process}

Biosorption and biodegradation of repeated-batch feed of
Table 1 Orthogonal experiments of concentrations of carbon source and nitrogen source, and the incubated spore suspension on bio-dissipation of PAHs by $P$. chrysosporium

\begin{tabular}{ccccc}
\hline Group & \multicolumn{3}{c}{ Influencing factor } & $\begin{array}{c}\text { biomass }{ }^{\mathrm{a}} \text { ) } \\
(\mathrm{mg})\end{array}$ \\
\cline { 2 - 4 } & $\begin{array}{c}\mathrm{NaNO}_{3} \\
(\mathrm{mmol} / \mathrm{L})\end{array}$ & $\begin{array}{c}\text { Glucouse } \\
(\mathrm{mmol} / \mathrm{L})\end{array}$ & $\begin{array}{c}\text { Spore suspension } \\
(\text { ceus/mL) }\end{array}$ & 3.54 \\
2 & 2.4 & 5.6 & $10^{5}$ & 3.44 \\
3 & 2.4 & 5.6 & $10^{6}$ & 15.98 \\
4 & 2.4 & 56 & $10^{5}$ & 16.23 \\
5 & 2.4 & 56 & $10^{6}$ & 4.63 \\
6 & 24 & 5.6 & $10^{5}$ & 5.14 \\
7 & 24 & 5.6 & $10^{6}$ & 17.48 \\
8 & 24 & 56 & $10^{5}$ & 17.92 \\
\hline
\end{tabular}

a) The dry weight of biomass after a 60-d incubation.

Phen under different concentrations of carbon source, nitrogen source and co-existing $\mathrm{Cu}^{2+}$ were studied. Eight groups were designed as follows: a heat-killed mycelial pellet $+\mathrm{NaN}_{3}$ as a bacteriostat (Group A), a heat-killed mycelial pellet $+0.25 \mathrm{mmol} / \mathrm{L} \mathrm{CuCl}_{2}$ (Group B), a live mycelial pellet (Group C), a live mycelial pellet $+56 \mathrm{mmol} / \mathrm{L}$ glucose (Group D), a live mycelial pellet $+2.4 \mathrm{mmol} / \mathrm{L}$ $\mathrm{NaNO}_{3}$ (Group E), a live mycelial pellet $+56 \mathrm{mmol} / \mathrm{L}$ glucose $+2.4 \mathrm{mmol} / \mathrm{L} \mathrm{NaNO}_{3}$ (Group F), a live mycelial pellet $+0.25 \mathrm{mmol} / \mathrm{L} \mathrm{CuCl}_{2}$ (Group G), a live mycelial pellet +56 $\mathrm{mmol} / \mathrm{L}$ glucose $+0.25 \mathrm{mmol} / \mathrm{L} \mathrm{CuCl}_{2}$ (Group $\mathrm{H}$ ). The experiment was conducted in $40-\mathrm{mL}$ vials. In addition of $15-\mathrm{mL} 1.0 \mathrm{~mol} / \mathrm{L}$ Phen, the vials were sealed with aluminum foil-lined Teflon screw caps (except the group containing $\mathrm{CuCl}_{2}$ in case the occurrence of the displacement reaction, and this operation has no effect on the experimental results) to avoid evaporation, and then agitated in the dark at $35 \pm 0.5^{\circ} \mathrm{C}$ with aeration for $2 \mathrm{~min}$ each day. After each $6 \mathrm{~d}$ of incubation, 3 parallels were taken from all Groups A-H to measure the residual concentrations of Phen in solution and mycelial pellets, and the dry weight of biomass. Repeat batch operation was handled for the rest parallels. The solution in the vials was decanted as much as possible, and then added $15-\mathrm{mL} 1.0 \mathrm{~mol} / \mathrm{L}$ Phen and the corresponding influencing factors again. A total of 5 cycles was conducted. The residual concentrations of Phen and the dry weight of biomass were detected at the incubation time of 6-, 12-, 18-, 24-, and 30-d in the repeat batch process. A group including only a live mycelial pellet without any influencing factor (CK) was set to measure the biosorption and biodegradation in the condition of continuous incubation without repeat batch operation. The single removal percentage, single degradation percentage and single adsorption capacity were calculated for each batch, in which the single removal percentage for each batch $\left(R_{x}\right)$ was calculated by

$$
R_{x}=\left(C_{0 x}-C_{x}\right) / \mathrm{C}_{0 x} \times 100 \% \text {, }
$$

where $x$ is the number of batches, $C_{0 x}(\mu \mathrm{g} / \mathrm{mL})$ is the initial 
concentration of Phen in the $x$ batch, $C_{x}(\mu \mathrm{g} / \mathrm{mL})$ is the concentration of Phen after a 6-d of incabation in the $x$ batch. The single adsorption capacity (the residual quantity) was calculated by the mass of sorbed Phen minus the mass in the previous batch. The positive single adsorption capacity meant that the Phen in solution was removed by biosorption while the negative value suggested that the sorbed-Phen was dissolved into solution or bio-degraded. The value of single degradation is detected by the mass balance calculation, which is ratio of Phen that bio-degraded to the dosage of Phen in the corresponding batch.

\section{Results and discussion}

\subsection{Biosorption and biodegradation of Phen and Pyr by fungi $P$. chrysosporium}

The removal of Phen and Pyr in solution by dead or live bodies of $P$. chrysosporium are shown in Figure 1. The biosorption of Phen and Pyr by heat-killed mycelial pellets reached apparent equilibrium within $3 \mathrm{~d}$, and $40.23 \% \pm$ $0.11 \%$ of Phen and $74.71 \% \pm 0.60 \%$ of Pyr were removed from solution. It is reported that biosorption mechanism of nonpolar organic pollutants by dead biomass is partition $[15,22]$. The respective partition coefficients of phenanthrene and pyrene are 4040 and $17500 \mathrm{~L} / \mathrm{kg}$. The sorption properties are similar to the white-rot fungi isolated from wood waste [22], of which the $K_{\mathrm{p}}$ values are $3721 \mathrm{~L} / \mathrm{kg}$ for Phen and $15980 \mathrm{~L} / \mathrm{kg}$ for Pyr, respectively. The adsorptive capacity of Pyr was higher than Phen, which is in line with their $K_{\mathrm{ow}}$ values. The removal of PAHs by heat-killed fungi kept constant at the incubation time from 3 to 40 -d, indicating biosorption is the only mechanism to remove PAHs from solution. The volatilization losses were small, ranking at $4.17 \% \pm 0.11 \%$ for Phen and at $3.55 \% \pm 0.31 \%$ for Pyr.

The removal percentages of Phen by live and heat-killed mycelial pellets were similar at the incubation time of 3-d which were $40.11 \%$ for live mycelial pellets and $40.23 \%$ for heat-killed mycelial pellets. While the removal percentage of Pyr by live mycelial pellets was $67.76 \%$ in $3-d$, lower than that of the heat-killed mycelial pellets $(74.71 \%)$. The removal of Phen and Pyr by live mycelial pellets increased with the extending incubation time, and the removal percentage reached $74.44 \%$ (Phen) and $86.24 \%$ (Pyr) at the incubation time of 40-d, which were obviously higher than the removal percentage in groups containing only heatkilled mycelial pellets. Both biosorption and biodegradation were contributed to the dissipation of PAHs by live $P$. chrysosporium in water. After a 3-d incubation, the biosorption percentages by live $P$. chrysosporium were $19.71 \%$ and $52.21 \%$ for Phen and Pyr, respectively, which are significantly lower than that of dead fungal biomass. This is mainly attributed to the change in $P$. chrysosporium cell permeability under the high-pressure inactivation treatment [34]. With the increase of the incubation time (3-40 d), biodegradation percentages gradually increased from $20.40 \%$ to $60.62 \%$ for Phen and from $15.55 \%$ to $49.21 \%$ for Pyr, and the stored-PAHs in the fungal bodies gradually decreased by $5.89 \%$ for Phen and by $15.18 \%$ for Pyr at $40-d$, which suggested that PAHs both in solution and fungal body can be biodegraded. The degradation process is consistent with the biosorption behavior of fungal body, which was controlled by partition and the process of sorption-desorption was reversible $[15,22]$.

Biosorption of organic pollutants by microbial body is a common phenomenon in the environment. Microbial remediation is one of the most potential technologies for PAHs abatement [12,13]. However, biosorption of PAHs by

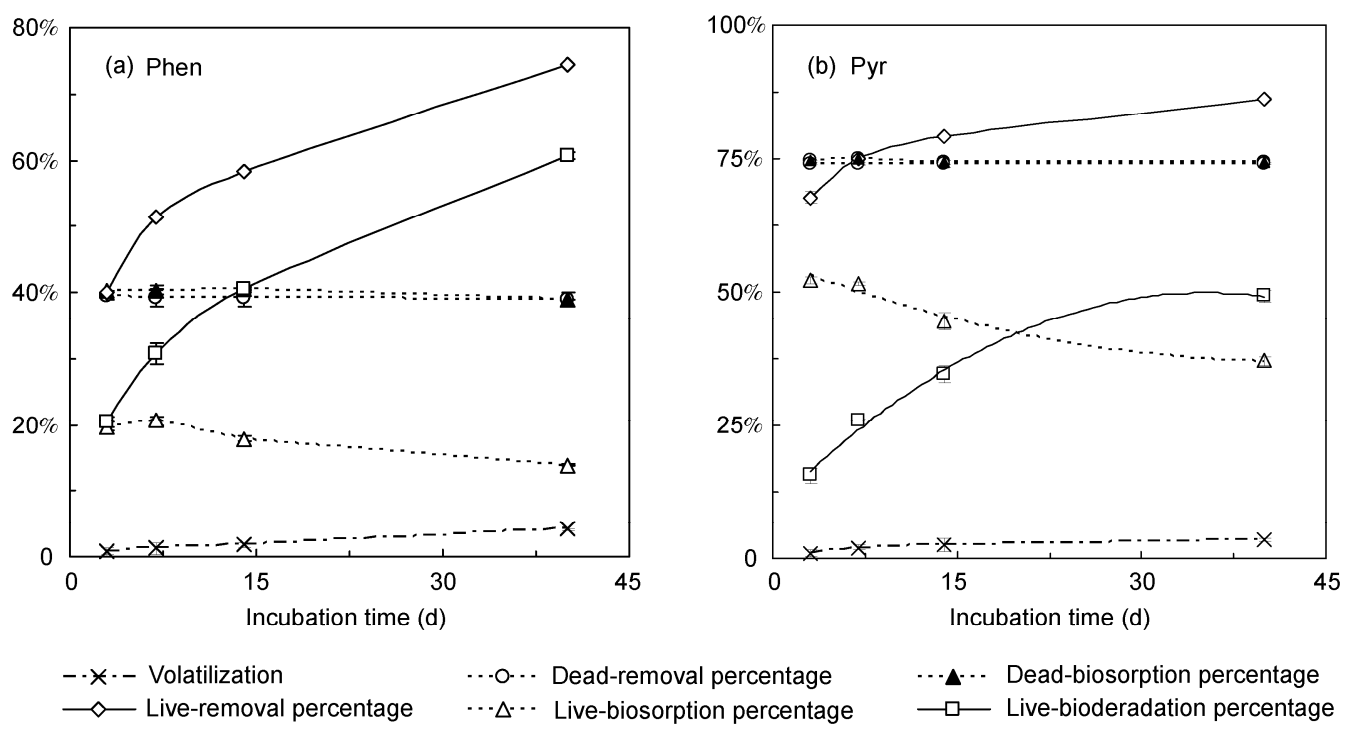

Figure 1 Biosorption and biodegradation of Phen (a) and Pyr (b) by P. chrysosporium cultivated under nutrient-free condition. 
microorganisms has been ignored in bioremediation practices. In fact, biosorption obviously contributes to the apparent removal of PAHs, which result in PAHs accumulation in microbial body rather than dissolved in solution [15]. For example, $19.71 \%$ of Phen and $52.21 \%$ Pyr were removed from solution by biosorption of Pc mycelial pellets within $3 \mathrm{~d}$ in the current study. The growth of mycelial pellets can be ignored because no any nutrient element or microelement was added. The average dry weight of live mycelial pellets was $2.22 \pm 0.05 \mathrm{mg}$, which was similar to that of the heat- killed mycelial pellets $(2.25 \pm 0.12 \mathrm{mg})$. However, the apparent removal in live mycelial pellets group was higher because of biodegradation, and $60.62 \%$ of Phen and $49.21 \%$ of Pyr was removed from the system by biodegradation.

\subsection{The effect of nutritional conditions on biosorption and biodegradation of PAHs}

The biosorption and biodegradation of Phen and Pyr under
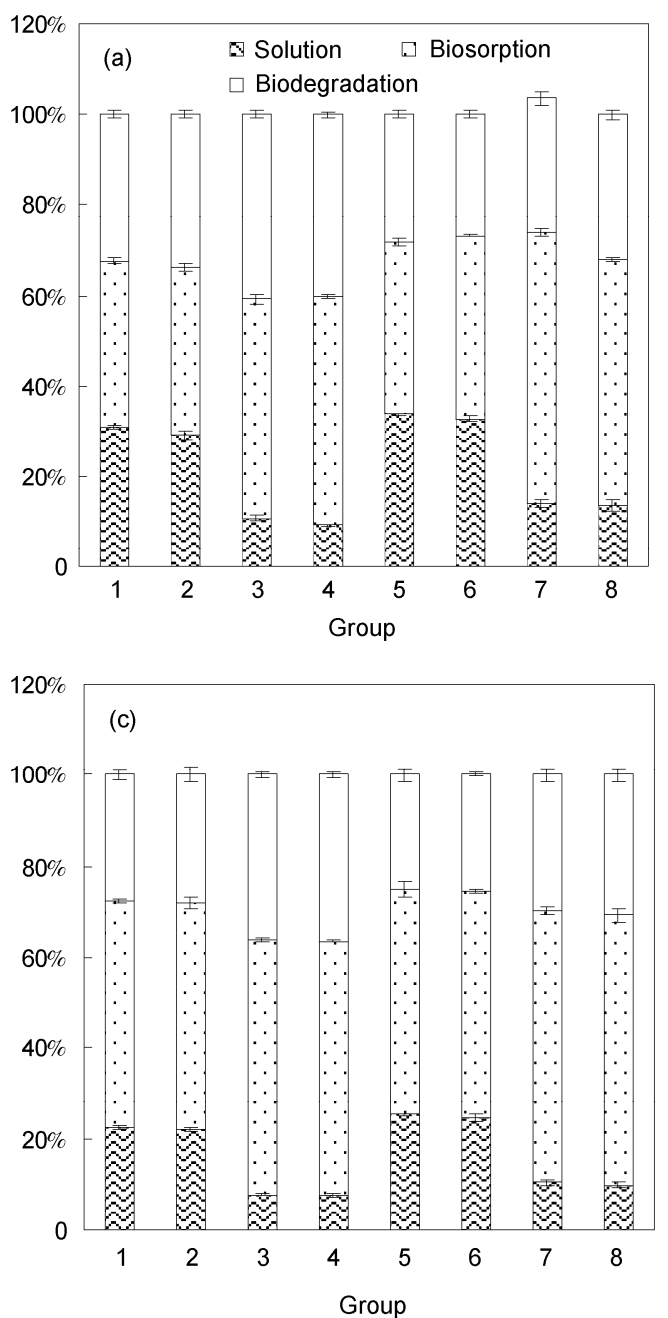

different concentration of carbon source and nitrogen source are shown in Figure 2. Both biosorption and biodegradation contributed to the removal of PAHs. Under the nutrient condition of carbon-rich (56 mmol/L glucose) and nitrogenrich $\left(24 \mathrm{mmol} / \mathrm{L} \mathrm{NaNO}_{3}\right)$ and inoculated with $10^{6}$ ceus $/ \mathrm{mL}$ spores (Group 8), 54.25\% of Phen and 59.30\% of Pyr were removed by biosorption while $32.16 \%$ of Phen and $30.85 \%$ of Pyr was removed by biodegradation within $10 \mathrm{~d}$. At the incubation time of $60-\mathrm{d}$, most of PAHs were removed by biodegradation, and the biodegradation percentage was 84.77\% for Phen and $77.96 \%$ for Pyr. The residual PAHs existed as bio-sorbed PAHs while little was free in the solution. Carbon source was an important influential factor on biosorption of Phen and Pyr. As the carbon-rich condition contributed to the growth of fungi (Table 1), the removal percentages in the groups (Groups 3, 4, 7 and 8) were obviously higher than that in the other groups (Figure 2). Both biosorption and biodegradation were improved with the growth of $P$. chrysosporium. Analysis of variance found that the $F$ values of nitrogen concentration and carbon
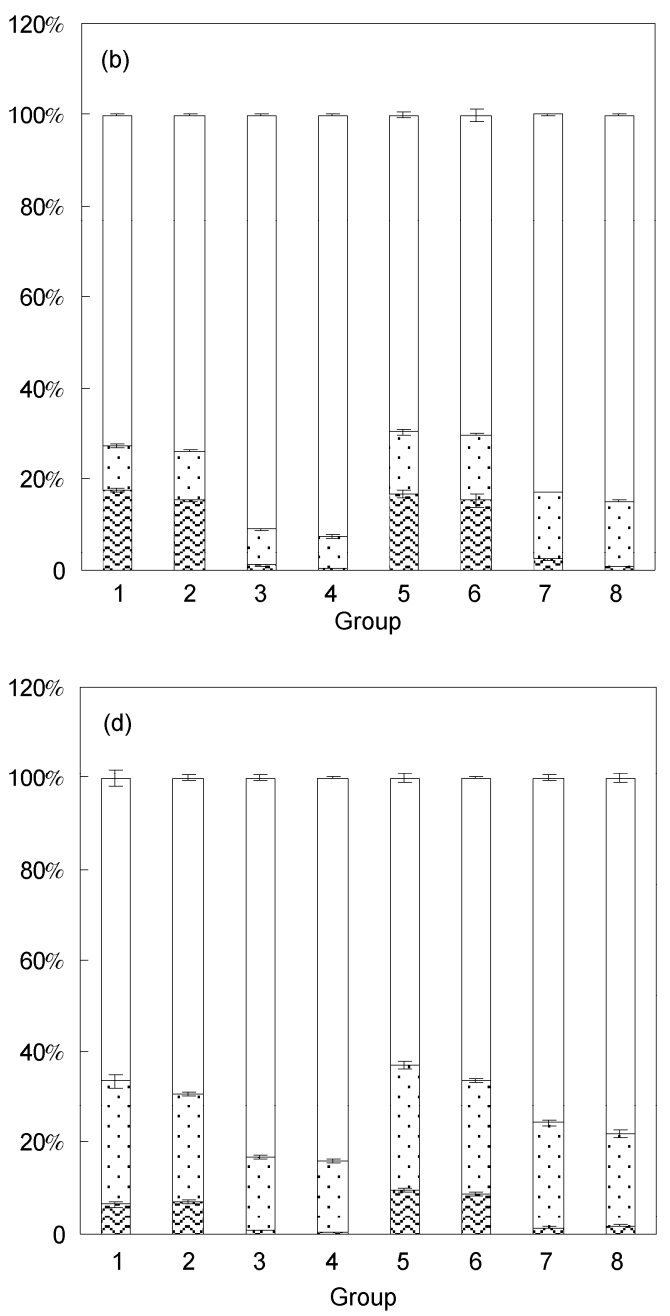

Figure 2 Biodissipation, biosorption and biodegradation by live Phanerochaete chrysosporium under different nutrient conditions. Phenanthrene with (a) 10-d and (b) 60-d incubation. Pyrene with (c) 10-d and (d) 60-d incubation. Error bars at the top of each column. See Table 1 for the nutrient condition of Groups 1-8. 
concentration effects on the biomass were 119.89 and 8672.57 , suggesting both of them had significant impact on the growth of $P$. chrysosporium while the carbon source was the controlling factor.

Although PAHs in solution can be removed by both biosorption and biodegradation, biosorption was a process that PAHs were pre-accumulated on a solid phase while biodegradation contributed to the completely elimination of organics. Thus, it is necessary to identify the relative contribution of biosorption and biodegradation to precisely predict fate of POPs in the environment and optimizing bioremediation practices. The orthogonal analysis of data is shown in Table 2. It is clear that carbon source and nitrogen source play a combined effect on biosorption and biodegradation. The carbon source had significant effect on the removal of Phen because carbon source was the controlling factor on biomass of $P$. chrysosporium. The effect of nitrogen concentration was much more significant in the shortterm $(10 \mathrm{~d})$ than in the long-term culture $(60 \mathrm{~d})$. In addition to controlling the growth of fungal body, the nitrogenlimited condition induced the biodegradation of PAHs in the short-term of incubation [33,35]. The removal percentages of Phen in Group 4 (carbon-rich, nitrogen-limit, $10^{6}$ ceus/ $\mathrm{mL}$ spores) and Group 8 (carbon-rich, nitrogen-rich, $10^{6}$ ceus $/ \mathrm{mL}$ spores) were $90.83 \%$ and $86.41 \%$ at the incubation time of $10 \mathrm{~d}$, and the percentages were $99.55 \%$ and $99.12 \%$ at the incubation time of $60-\mathrm{d}$, respectively. The Group 4 was the most effective system to remove PAHs from solution.

Table 2 Orthogonal analysis of the influence of concentration of carbon source, nitrogen source and the incubated spore suspension on the bio-dissipation, biosorption, biodegradation of phenanthrene ${ }^{\text {a) }}$

\begin{tabular}{|c|c|c|c|c|c|}
\hline & \multirow{2}{*}{$\begin{array}{l}\text { Variance } \\
\text { sources }\end{array}$} & \multicolumn{2}{|r|}{$10 \mathrm{~d}$} & \multicolumn{2}{|r|}{$60 d$} \\
\hline & & $F$ value & Significance & $F$ value & Significance \\
\hline \multirow{4}{*}{ Removal } & A(nitrogen) & 174.9 & $* * *$ & 0.87 & \\
\hline & B(carbon) & 5144 & $* * *$ & 1969 & $* * *$ \\
\hline & $A^{*} B$ & 0.70 & & 4.53 & \\
\hline & C(spores) & 19.17 & $* *$ & 19.79 & $* *$ \\
\hline \multirow{4}{*}{ Biosorption } & A(nitrogen) & 10.38 & $* *$ & 155.9 & $* * *$ \\
\hline & B(carbon) & 155.4 & $* * *$ & 10.83 & $* *$ \\
\hline & $A^{*} B$ & 2.067 & & 20.58 & $* *$ \\
\hline & C(spores) & 0.63 & & 0.49 & \\
\hline \multirow{4}{*}{ Biodegradation } & A(nitrogen) & 96.48 & $* * *$ & 173.3 & $* * *$ \\
\hline & B(carbon) & 55.03 & $* * *$ & 1580 & $* * *$ \\
\hline & $\mathrm{A} * \mathrm{~B}$ & 6.29 & $*$ & 37.38 & $* * *$ \\
\hline & C(spores) & 0.16 & & 17.19 & $* *$ \\
\hline
\end{tabular}

a) In all analysis of variance table, the sum of squares $(S)$ of $\mathrm{A} * \mathrm{C}$ and $\mathrm{A} * \mathrm{~B}$ was smaller than error column, means the the interaction between $\mathrm{A}$ and $\mathrm{C}, \mathrm{B}$ and $\mathrm{C}$ can be neglected. $F_{0.01}(1,3)=34.12, F_{0.05}(1,3)=10.13$, $F_{0.1}(1,3)=5.54$. * indicates $F_{0.05}>F>F_{0.1}$, influential but not significantly; ** indicates $F_{0.01}>F>F_{0.05}$, significantly influenced; *** indicates $F>$ $F_{0.01}$, very significantly influenced.
As shown in Table 2, concentrations of carbon source and nitrogen source exhibited a complex effect on biodegradation of $P$. chrysosporium, in which the $F$ value of nitrogen source (96.47) was higher than the value of carbon source (55.03) in the short-term (10 d), supporting the induction of nitrogen source to biodegradation. Compared the Group 4 (carbon-rich, nitrogen-limit) and Group 8 (carbon-rich, nitrogen-rich), although the apparent removal percentage was similar, but the biodegradation percentage in Group $4(92.77 \%)$ was higher than the percentage in Group 8 (82.05\%), indicating more Phen was eliminated by biodegradation under the nutrient condition of nitrogenlimit while more Phen was accumulated into the biomass (biosorption) under the nutrient condition of nitrogen-rich. In the tested groups, the biodegradation percentage in Group 4 was the highest. A similar conclusion was gotten by analyzing the data of Pyr.

\subsection{Biosorption and biodegradation of Phen by $P$. chrysosporium in the repeated batch process}

Pre-cultured mycelial pellets were used in the experiment in order to induce enough biomass quickly and enhance the ability of fungi to adapt the test condition [24]. The biosorption and biodegradation of $P$. chrysosporium in the repeated batch process are shown in Figure 3 . The single removal percentage of Phen by heat-killed mycelial pellets gradually decreased in the repeated batch process. The removal percentage of Phen by dead mycelial pellets was $38.69 \% \pm$ $0.76 \%$ in the first batch, while after 5-cycle, only $0.79 \% \pm$ $0.36 \%$ of Phen was removed. It was reported that co-existing of $\mathrm{Cu}^{2+}$ increased the biosorption of PAHs [22]. Compared Groups A and B in Figure 3, the co-existing of $\mathrm{Cu}^{2+}$ increased the biosorption of Phen in the repeated batch process but the effect was not so obvious. In the first batch, $38.69 \%$ and $40.77 \%$ of Phen was removed in Group A without $\mathrm{Cu}^{2+}$ and Group $\mathrm{B}$ containing $\mathrm{Cu}^{2+}$, respectively; while after 5-cycle, only $0.79 \%$ and $2.26 \%$ of Phen was removed in Groups A and B. Thus, the dead microorganism is not capable to remove organics in the repeated batch process. To meet the need of removal POPs, a large amount of sludge would be produced, and then caused POPs secondary pollution after the sludge discharged.

As shown in Figure 3(b), the single removal percentage of live mycelial pellets without augmentation of nutrition (Group C) decreased in the repeated batch process, i.e. $62.35 \%$ for the 1 st batch and $24.06 \%$ for the 5 th batch, which was still higher than the removal percentage by dead mycelial pellets (Group A, i.e. 38.69\% and 0.79). As shown in Figure 3(a), the dry weight of live mycelial pellets under no nutritional conditions was similar to the dead pellets (Groups A and B), suggesting biodegradation contributed to the increase of removal percentage. The dashed line in Figure 3(c) indicated the initial biodegradability of $P$. chrysosporium under no nutritional conditions. And the biodegra- 
dation percentage was higher than the percentage of in 1st batch, indicating the increase of biodegradability. Reasonably, with the increase of cycle times, the apparent removal percentage decreased was attributed to the decline in the biosorption for Group C.

The influence of carbon source, nitrogen source and $\mathrm{Cu}^{2+}$ on the bisorption and biodegradation in the repeated batch process were studied in Groups D-H. The single removal percentage of Phen in Group F (carbon-rich, nitrogen-limit) was the highest in all experimental groups, which maintained $>81 \%$ in all the 5 cycles and reached $89.74 \%$ in the 5 th batch, suggesting augmentation of carbon and nitrogen source increased the removal of PAHs in the repeated feed batch process. Under the nutrient condition of carbon-rich, the biomass of fungi increased significantly, i.e. the dry weight of mycelial pellets increased to $13.44 \pm 0.38 \mathrm{mg}$ with the augmentation of carbon-rich and nitrogen-limit (Group F) at the incubation time of $60 \mathrm{~d}$ compared with the initial weight about $2.22 \mathrm{mg}$. On the other hand, the augmentation of carbon-rich and nitrogen-limit enhanced the biodegradation. The single biodegradation percentage in Group $F$ gradually improved from 1 st to 3 rd batch and keeping
$>90 \%$ after 3rd batch (Figure 3(c)). This meant that $>90 \%$ of fresh Phen was eliminated by biodegradation in each batch after 3 cycles, so as to achieve stable removal of PAHs. In group with live mycelial pellets, the Phen in solution as well as the sorbed-PAHs on the mycelial pellets decreased. As shown in Figure 3(d), the single adsorption capacity (the residual quantity) was negative after 3 cycles, implying the residual Phen on mycelial pellets decreased although continuous feed of fresh Phen, which ensure the low residual of Phen during the repeated batch process. The residual Phen on the pellets of Group C (without augmentation) decreased in 5th batch, suggesting biodegradation played an important role in the removal of PAHs by live fungi, and result in a higher removal percentage comparing with the groups containing heat-killed mycelial pellets only. Keyser et al. [33] divided the life cycle of $P$. chrysosporium into the growing season, the breeding season and the decline phase, and the secondary metabolic processes of pollutants (such as PAHs) happened in the breeding season. The continuous feed of fresh PAHs, carbon source and nitrogen source contributed to maintain the secondary metabolism, thus the biodegradation kept high during the experimental
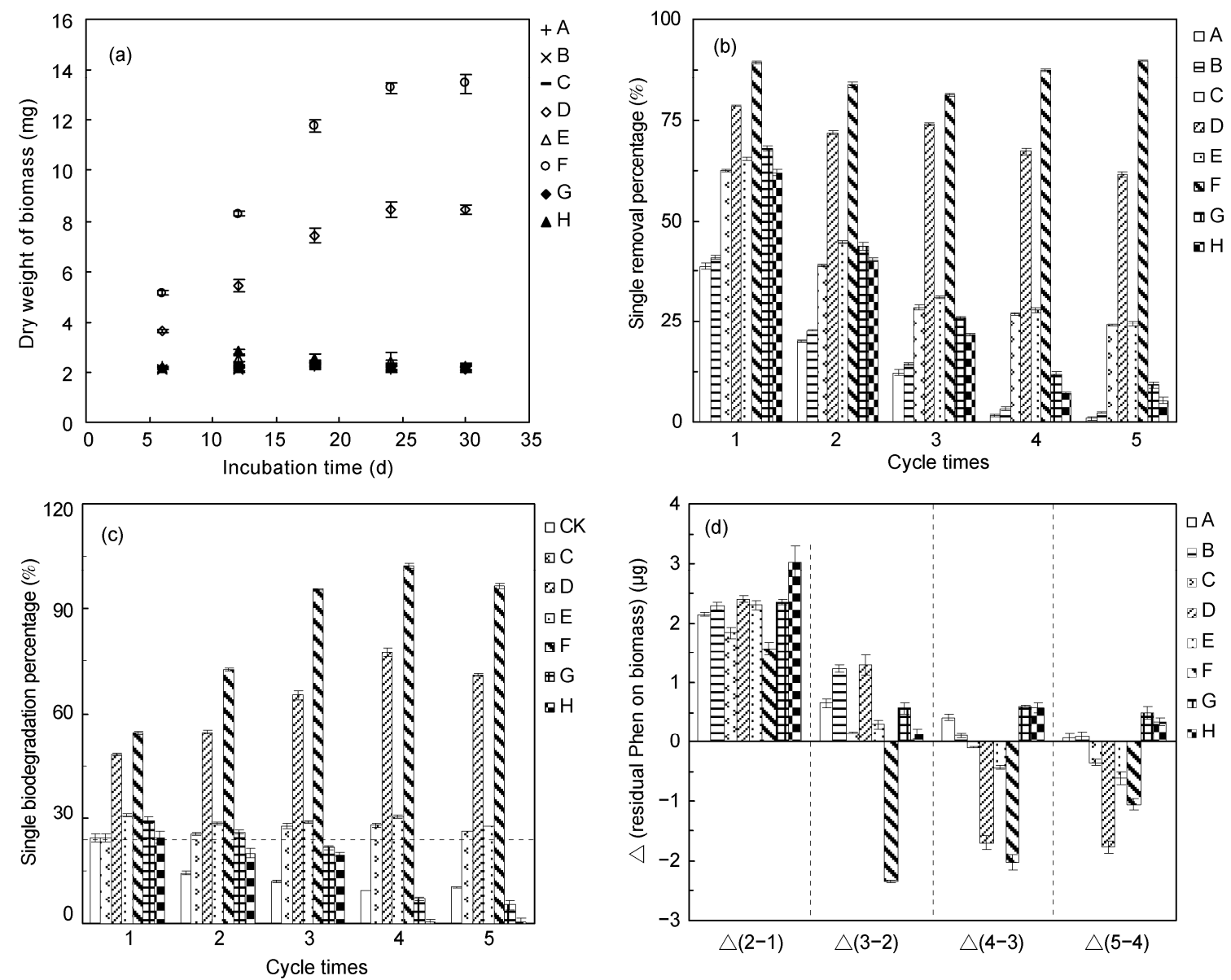

Figure 3 Biosorption and biodegradation of phenanthrene by Phanerochaete chrysosporium in the repeated batch process. (a) The mass of Pc biomass; (b) the total removal of phenanthrene in solution in each sequential batch; (c) the biodegradation of phenanthrene in each sequential batch; (d) the difference of phenanthrene biosorption between every two sequential batch. 


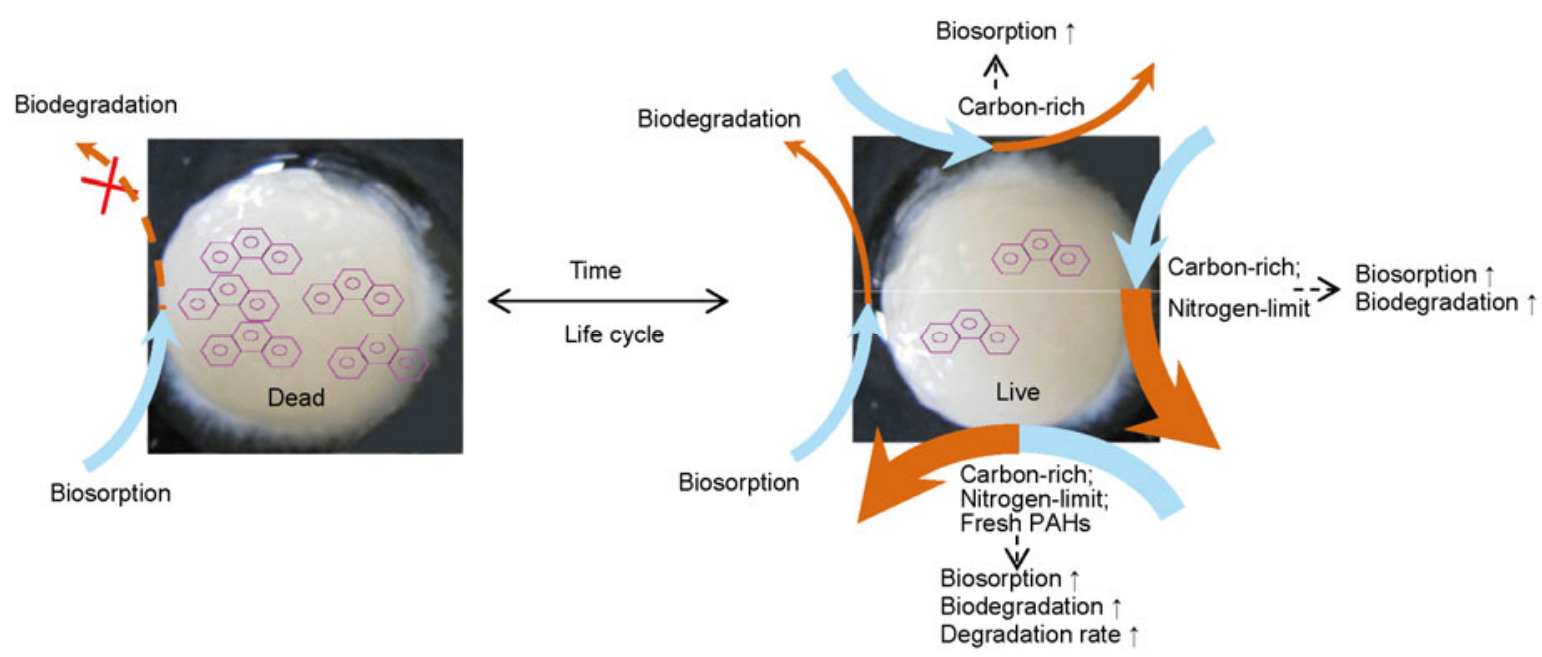

Figure 4 Scheme of biosorption and biodegradation of polycyclic aromatic hydrocarbons by dead/live Phanerochaete chrysosporium and their regulating factors.

period of $30 \mathrm{~d}$.

The co-existing of $\mathrm{Cu}^{2+}$ not only improved the biosorption of Phen, but also enhanced the biodegradation (Group $\mathrm{G})$. In the 1st batch, the biodegradation percentage in Group $\mathrm{G}$ which contained live mycelial pellets and $\mathrm{Cu}^{2+}$ was $29.29 \% \pm 0.96 \%$, higher than the biodegradation in group without $\mathrm{Cu}^{2+}(24.28 \% \pm 1.02 \%)$. But in the repeated batch process, $\mathrm{Cu}^{2+}$ was accumulated on the fungal body, thus the growth of fungi was inhibited and the biomass did not increase. Therefore, the contribution of biodegradation decreased gradually. In the 5th batch, the biodegradation percentages in group with/without $\mathrm{Cu}^{2+}$ were $5.12 \%$ and $25.20 \%$. The toxicity of $\mathrm{Cu}^{2+}$ to $P$. chrysosporium was greater with augmentation of carbon-rich. The biodegradation percentage of Group $\mathrm{H}$ containing both carbon-rich and $\mathrm{Cu}^{2+}$ was only $0.25 \%$ in the 5 th batch. The co-existing of $\mathrm{Cu}^{2+}$ improved both biosorption and biodegradation, but biodegradation decreased with cycles, thus, $\mathrm{Cu}^{2+}$ can not be an augmentation factor for the repeated batch process.

\subsection{The regulating principle of biosorption and bio- degradation of $P$. chrysosporium}

The schematic diagram of the biosorption and biodegradation of dead/live $P$. chrysosporium mycelial pellets and their regulating factors are shown in Figure 4. The dead mycelial pellets removed PAHs by biosorption only and the fungal biomass can be considered as a bio-sorbent. The biosorption of live fungi can be enhanced with augmentation of carbon-rich to reduce the PAHs in solution immediately, while biosorption and biodegradation can be both improved with augmentation of carbon-rich and nitrogen-limit and result in low residual of PAHs in solution as well as on biomass. Moreover, the degradation rate is promoted when augmentation of carbon-rich, nitrogen-limit and continuous feed of fresh PAHs. Therefore, it is feasible to regulate biosorption and biodegradation contribution in bioremediation, and to provide a theoretical gist for sewage activated sludge treatment system to remove POPs rapidly and efficiently.

\section{Conclusions}

(1) Both biosorption and biodegradation of P. chrysosporium contributed to the removal of Phen and Pyr in solution. Biosorption favored the rapid transfer of PAHs from solution to fungal biomass, while biodegradation contributed to the dissipation of PAHs in solution and in fungal body, and biodegradation increased with the incubation time.

(2) Under the nutrient condition of carbon-rich and nitrogen-limit, the removal efficiency and biodegradation percentage of Phen and Pyr were significantly promoted, i.e. 99.55\% and $92.77 \%$ for Phen, and $99.47 \%$ and $83.97 \%$ Pyr after 60-d incubation. This phenomenon was ascribed to enhanced-biosorption due to the increase of fungal biomass under the carbon-rich condition, and to stimulated-biodegradation under the nitrogen-limit condition.

(3) In the repeated batch process, the removal of PAHs by live mycelial pellets showed higher than the dead pellets. The biodegradation percentage of Phen kept $>81 \%$ in all 5 cycles with the augmentation of carbon-rich and nitrogenlimit conditions and after 3 cycles, the biodegradation percentage was up to $90 \%$ with each cycle of 6-d incubation. The low residual of PAHs on biomass ensured the continuous process running.

This work was supported by the National Natural Science Foundation of China (41071210), the National High Technology Research and Development Program of China (2012AA06A203) and Zhejiang Provincial Natural Science Foundation of China (R5100105).

1 Yao Z W, Jiang G B, Cai Y Q, et al. Status of persistent organic pol- 
lutants and heavy metals in surface water of Arctic region. Chin Sci Bull, 2003, 48: 131-135

2 Breivik K, Alcock R, Li Y F, et al. Primary sources of selected POPs: Regional and global scale emission inventories. Environ Pollut, 2004, 128: 3-16

3 Lohmann R, Breivk K, Dachs J, et al. Global fate of POPs: Current and future research directions. Environ Pollut, 2007, 150: 150-165

4 Chen B L, Xuan X D, Zhu L Z, et al. Distributions of polycyclic aromatic hydrocarbons in surface waters, sediments and soils of Hangzhou city, China. Water Res, 2004, 38: 3558-3568

5 Wang X P, Yao T D, Cong Z Y, et al. Concentration level and distribution of polycyclic aromatic hydrocarbons in soil and grass around Mt. Qomolangma, China. Chin Sci Bull, 2007, 52: 1405-1413

6 Zhang Y X, Tao S, Shen H Z, et al. Inhalation exposure to ambient polycyclic aromatic hydrocarbons and lung cancer risk of Chinese population. Proc Natl Acad Sci USA, 2009, 106: 21063-21067

$7 \mathrm{Ru}$ J, Liu H J, Qu J H, et al. Characterization and adsorption behavior of a novel triolein-embedded activated carbon composite adsorbent. Chin Sci Bull, 2005, 50: 2788-2790

8 Chen B L, Zhou D D, Zhu L Z, et al. Sorption characteristics and mechanisms of organic contaminant to carbonaceous biosorbents in aqueous solution. Sci China Ser B-Chem, 2008, 51: 464-472

9 Nizzetto L, Macleod M, Borga K, et al. Past, present, and future controls on levels of persistent organic pollutants in the global environment. Environ Sci Technol, 2010, 44: 6526-6531

10 Chen B L, Yuan M X. Enhanced dissipation of polycyclic aromatic hydrocarbons in the presence of fresh plant residues and their extracts. Environ Pollut, 2012, 161: 199-205

11 Semple K T, Doick K J, Jones K C, et al. Defining bioavailability and bioaccessibility of contaminated soil and sediment is complicated. Environ Sci Technol, 2004, 38: 228A-231A

12 Bamforth S M, Singleton L. Bioremediation of polycyclic aromatic hydrocarbons: Current knowledge and future directions. J Chem Technol Biotechnol, 2005, 80: 723-736

13 Haritash A K, Kaushik C P. Biodegradation aspects of polycyclic aromatic hydrocarbons (PAHs): A review. J Hazard Mater, 2009, 169: $1-15$

14 Seo J S, Keum Y S, Li Q X. Bacterial degradation of aromatic compounds. Int J Environ Res Public Health, 2009, 6: 278-309

15 Chen B L, Wang Y S, Hu D H. Biosorption and biodegradation of polycyclic aromatic hydrocarbons in aqueous solutions by a consortium of white-rot fungi. J Hazard Mater, 2010, 179: 845-851

16 Chan S M N, Luan T, Wong M, et al. Removal and biodegradation polycyclic aromatic hydrocarbons by Selenastrum capricornutum. Environ Toxicol Chem, 2006, 25: 1772-1779

17 Vijayaraghavan K, Yun Y S. Bacterial biosorbents and biosorption. Biotechnol Adv, 2008, 263: 266-291

18 Liu Y Y, Fu J C, Hu H B, et al. Properties and characterization of $\mathrm{Au}^{3+}$-adsorption by mycelial waste of Streptomyces aureofaciences.
Chin Sci Bull, 2001, 46: 1709-1712

19 Gao D W, Wen X H, Qian Y. Decolorization of reactive brilliant red $\mathrm{K}-2 \mathrm{BP}$ with the white-rot fungi under non-sterile conditions. Chin Sci Bull, 2004, 49: 981-982

20 Aksu Z. Application of biosorption for the removal of organic pollutants: A review. Process Biochem, 2005, 40: 997-1026

21 Park D, Yun Y S, Park J M. The past, present, and future trends of biosorption. Biotechnol Bioproc Eng, 2010, 15: 86-102

22 Ding J, Wang Y S, Shen X Y, et al. Bioremediation tests on biosorption-desorption of phenanthrene and pyrene by white-rot fungi (in Chinese). Acta Scientiae Circumstantiae, 2010, 30: 825-831

23 Stringfellow W T, Alvarez-Cohen L. Evaluating the relationship between the sorption of PAHs to bacterial biomass and biodegradation. Water Res, 1999, 33: 2535-2544

24 Yesilada O, Yildirim S C, Birhanli E, et al. The evaluation of pregrown mycelia pellets in decolorization of textile dyes during repeated batch process. World J Microbiol Biotechnol, 2010, 26: 33-39

25 Bumpus J A, Tien M, Wright D, et al. Oxidation of persistent environmental-pollutants by a white rot fungus. Science, 1985, 228: 1434-1436

26 Kim D Y, Rhee Y H. Biodegradation of microbial and synthetic polyesters by fungi. Appl Microbiol Biotechnol, 2003, 61: 300-308

27 Wesenberg D, Kyriakides I, Agathos S N. White-rot fungi and their enzymes for the treatment of industrial dye effluents. Biotechnol Adv, 2003, 22: 161-187

28 Singh D, Chen S. The white-rot fungus Phanerochaete chrysosporium: Conditions for the production of lignin-degrading enzymes. Appl Microbiol Biotechnol, 2008, 81: 399-417

29 Barr D P, Aust S D. Mechanisms white-rot fungi use to degrade pollutants. Environ Sci Technol, 1994, 28: A78-A87

30 Loick N, Hobbs P J, Hale M D C, et al. Bioremediation of polyaromatic hydrocarbon (PAH)-contaminated soil by composting. Crit Rev Environ Sci Technol, 2009, 39: 271-332

31 Jeffries T W, Choi S, Krik T K. Nutritional regulation of lignin degradation by Phanerochaete chrysosporium. Appl Environ Microbiol, 1981, 42: 290-296

32 Schhimel J P, Weintraub M N. The implications of exoenzyme activity on microbial carbon and nitrogen limitation in soil: A theoretical model. Soil Biol Biochem, 2003, 35: 549-563

33 Keyser P, Kirk T K, Zeikus J G. Ligninolytic enzyme system of Phanerochaete chrysosporium: Synthesized in the absence of lignin in response to nitrogen starvation. J Bacteriol, 1978, 135: 790-797

34 Binupriya A R, Sathishkumar M, Kavitha D, et al. Experimental and isothermal studies on sorption of Congo Red by modified mycelial biomass of wood-rotting fungus. Clean, 2007, 35: 143-150

35 Dhawale S W, Dhawale S S, Deanross D. Degradation of phenanthrene by Phanerochaete-chrysosporium occurs under ligninolytic as well as nonligninolytic conditions. Appl Microbiol Biotechnol, 1992, 58: 3000-3006

Open Access This article is distributed under the terms of the Creative Commons Attribution License which permits any use, distribution, and reproduction in any medium, provided the original author(s) and source are credited. 